\title{
Rayonnement de la Faculté de foresterie et de géomatique sur la scène internationale
}

\author{
par
}

Jean Tomlinson ${ }^{1}$

\section{Résumé}

La Faculté de foresterie et de géomatique est une constituante importante de l'Université Laval. Installée dans la région de Québec depuis quelque 80 ans, cette Faculté intervient de façon active sur la scène internationale depuis plus de 20 ans. Ce document trace un portrait actuel des diverses facettes que prend cette intervention particulière. Les principales activités internationales de la Faculté sont ainsi regroupées en 10 volets différents. Ces dernières sont systématiquement définies, énumérées et au besoin quantifiées. Ce document permet de constater que la Coopération internationale fait partie intégrante de la mission fondamentale de la Faculté de foresterie et de géomatique, et que cette dernière veut s'y consacrer à la mesure de ses moyens.

\section{Abstract}

The Forestry and Geomatic School has always been an essential element of Laval University. Based in the Quebec City suburban area for about 80 years, the School has been active at the international level for more than 20 years. Thus, this paper presents the different aspects of this broad intervention. The main international activities of the School are divided into ten categories. These are listed, defined, and whenever needed, evaluated. The paper also demonstrates that the international cooperation is an important part of School basic mission and that we are willing to continue these activities as long as our means will permit.

\section{Introduction}

La Faculté de foresterie et de géomatique de l'Université Laval intervient sur la scène internationale depuis de nombreuses années et tracer un portrait actuel de cette intervention représente une tâche complexe. Les divers volets de cette participation internationale s'expriment en effet par des activités individuelles ou institutionnelles, tantôt ponctuelles tantôt soutenues. Grosso modo, I'histoire de cette implication facultaire comporte trois périodes: 1) avant 1970; 2) de 1970 à 1980, et 3) depuis 1981. Malgré l'intérêt que peuvent représenter l'ensemble des interventions effectuées jusqu'à aujourd'hui, ce document 'souligne d'abord l'état actuel du rayonnement de la Faculté de foresterie et de géomatique sur la scène internationale. II considère ses réalisations passées, d'abord comme fondement explicatif d'une certaine éclosion de sa coopération internationale et, ensuite, comme moyen de quantifier l'importance de cette dernière.

Ce document veut identifier et brièvement expliquer les diverses activités dont la portée est internationale et, lorsque possible, quantifier leur portée. Ces activités seront regroupées en dix (10) volets d'intervention internationale, soient:

\section{- Accords-cadres}

- Ententes particulières

- Étudiants non canadiens

'Université Laval, Cité Universitaire, Québec G1K 7 P4

Dans ce texte, le générique masculin est utilisé sans aucune discrimination et uniquement dans le but d'alléger le texte.
- Présentation de conférences

- Congrès et symposiums internationaux

- Missions techniques et professionnelles

- Organisation d'échanges

- Années sabbatiques ou sans solde

- Accueil de visiteurs non canadiens

- Autres volets d'intervention.

Il importe de mentionner dès le début la définition de coopération internationale: dans le contexte particulier de ce document, une activité de coopération internationale signifie toute activité officielle de la Faculté de foresterie et de géomatique ou de l'un de ses membres (qu'il soit professeur, chercheur, administrateur ou étudiant) réalisée dans, pour ou avec un pays autre que le Canada.

\section{Accords-cadres}

Dans de nombreux secteurs d'activités, I'Université Laval conclut des accords-cadres avec des universités, centres de recherches, instituts spécialisés ou associations professionnelles ou scientifiques, en vue de créer un climat propice à une éventuelle collaboration. Ces accords reflètent les intérêts communs et les volontés mutuelles des partenaires; très souvent, ils mentionnent, dès le départ, les domaines prioritaires visés. Un certain nombre de ces accords impliquent de façon toute particulière les domaines de la foresterie et de la géomatique; le tableau I les énumère et indique pour chacun d'eux les domaines visés. 


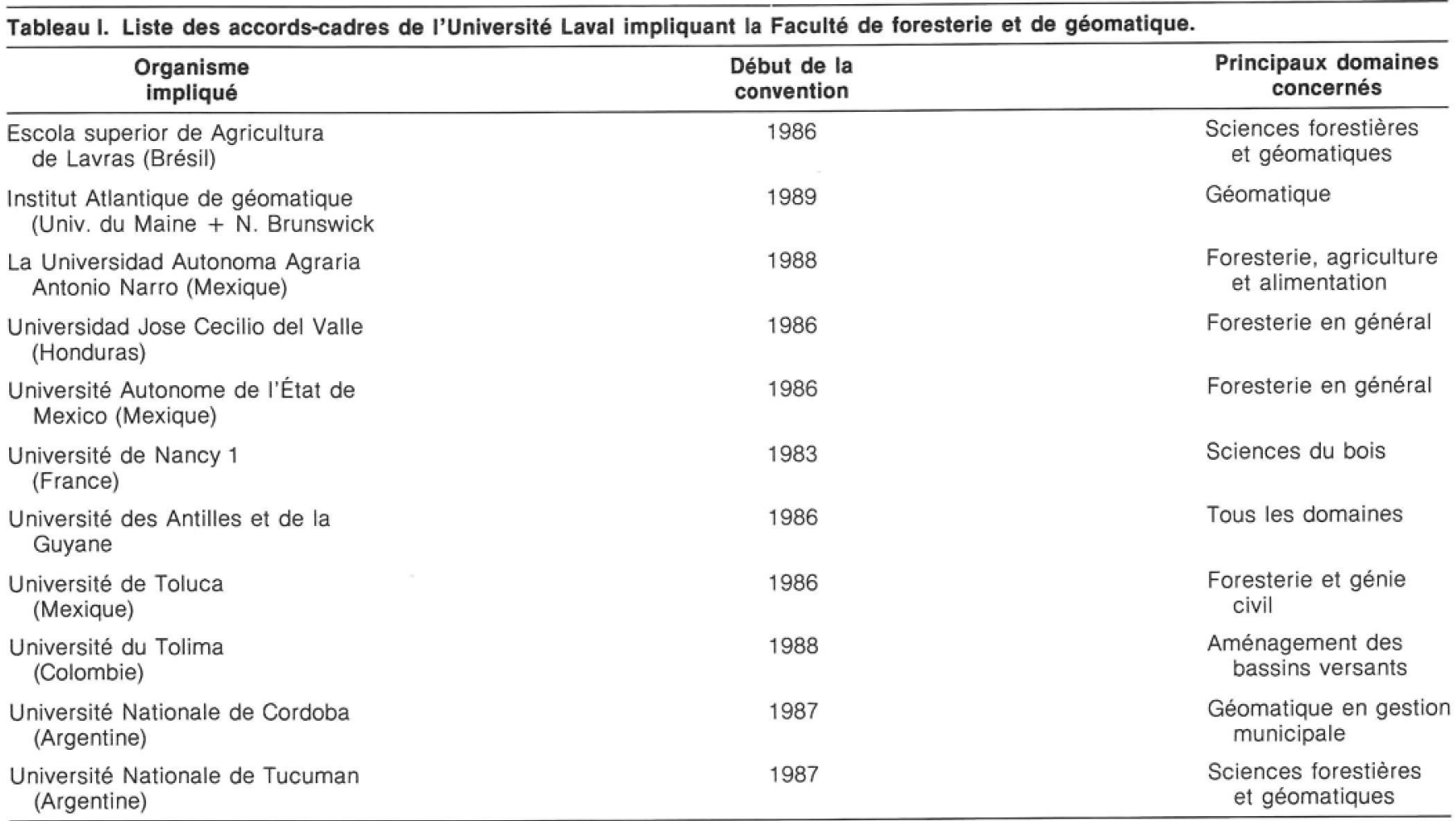

\section{Ententes particulières}

Que ce soit à l'intérieur de ces accords-cadres ou pas, la Faculté de foresterie et de géomatique a de plus établi des ententes particulières dont le déroulement repose sur l'existence de budgets spéciaux. Ce type de collaboration a, la plupart du temps, comme objectifs: la formation de professionnels forestiers ou de professeurs chercheurs. Les fonds nécessaires au fonctionnement de telles ententes proviennent actuellement de deux sources différentes: ACDI et CRDI.

Trois projets sont actuellement en cours, grâce à l'appui financier de l'ACDI; ces projets s'inscrivent dans le cadre d'ententes bi-latérales avec le Maroc, le Rwanda et le Zailre.

\section{Maroc: Institut agronomique et vétérinaire Hassan II}

Ce projet vise à renforcer la capacité du corps professoral des départements de topographie et de foresterie. L'entente actuelle (1986-1990) implique la formation au doctorat de six professeurs marocains ainsi que l'enseignement au Maroc de certaines matières spécialisées par des professeurs canadiens. Cette intervention est l'aboutissement d'une implication prolongée dans ce pays, initiée en 1972, et orientée alors uniquement vers les sciences géodésiques. II s'agit ici d'une entente institutionnelle convenue entre cet institut marocain et l'Université Laval, mais appuyée financièrement par I'ACDI.

\section{Rwanda : Appui institutionnel et formation forestière}

Ce projet vise à renforcer les capacités de gestion forestière du Rwanda. Quoique supporté financièrement par l'ACDI, le gouvernement québécois, via son Ministère des affaires internationales, en est l'agence d'exécution. L'Université Laval a, à son tour, conclu une entente spéciale (1987-1991) avec le gouvernement québécois pour ce qui concerne l'élément "formation". A l'intérieur d'une intervention globale d'appui institutionnel aux organismes rwandais de l'administration forestière par le gouvernement québécois, le volet "formation forestière" a comme objectif la formation de professionnels forestiers aptes à prendre la relève une fois terminée l'aide canadienne. Ainsi, les extrants du projet seront: trois candidats formés à la maîtrise, huit bacheliers spécialisés en sciences forestières ainsi que six techniciens formés en Côte d'Ivoire (Bouake). En plus des responsabilités liées à l'enseignement, cette entente implique pour la Faculté: le recrutement des candidats, un suivi pédagogique particulier, l'organisation de stages professionnels au Rwanda, la réalisation et l'encadrement de recherches locales pour les étudiants à la maîtrise, etc.

\section{Zaïre: Formation d'ingénieurs forestiers au Zaïre (Phase 2)}

Cette entente poursuit en quelque sorte les diverses interventions réalisées au Zaïre depuis 1970. La Faculté de foresterie et de géomatique participe en effet depuis cette date à la formation forestière dans ce pays, à un point tel qu'il n'est pas exagéré d'affirmer que $90 \%$ au moins de tous les professionnels forestiers zaïrois ont été formés sous sa responsabilité. Les modalités de cette formation ont changé avec les années mais les objectifs originaux sont toujours les mêmes, à savoir doter l'État zaïrois d'un personnel forestier compétent et diversifié. Le projet en cours de réalisation (1987-1992) comporte la formation de deux docteurs, de quatre diplômés de niveau maîtrise et de quelque 25 bacheliers spécialisés dans les divers domaines de la foresterie. En plus des responsabilités liées à l'enseignement, cette entente implique pour la Faculté comme au Rwanda: le recrutement des candidats, un suivi pédagogique particulier, l'organisation de stages professionnels au Zaïre, la réalisation et l'encadrement de recherches locales pour les étudiants à la maîtrise et au doctorat.

L'approche du CRDI est différente et découle directement de sa mission spécifique. L'objectif visé par son aide 
financière est la plupart du temps lié à la participation active des chercheurs des pays impliqués dans l'entente. Au cours des dernières années, la participation de la Faculté dans les projets appuyés par le CRDI a été concentrée dans 2 secteurs spécialisés, soit en biologie forestière et en télédétection.

\section{Le CRDI et la biologie forestiere}

Un des domaines d'excellence reconnus à l'Université Laval est celui de la fixation d'azote au moyen de champignons mycorhiziens. La Faculté de foresterie et de géomatique ayant acquis une renommée mondiale dans ce domaine, il était normal que ce domaine d'intervention intéresse un organisme comme le CRDI. Pour l'instant, l'implication de la Faculté consiste principalement à implanter, dans certaines régions du monde, des projets COOP. En bref, il s'agit d'aider à la mise sur pied de centres de recherche spécialisés dans le domaine d'excellence précité. Une telle initiative vise ultimement à favoriser le transfert technologique des connaissances, à former des chercheurs, à organiser des échanges de chercheurs, à faciliter l'installation de laboratoires et à collaborer à la définition de programmes de recherche. Les pays actuellement concernés par les projets COOP sont le Maroc, le Zaïre et I'Inde; d'autres pays pourraient s'ajouter à cette liste, la Chine par exemple. Au niveau facultaire, cette colloboration relève essentiellement du Centre de recherches en biologie forestière (CRBF).

\section{Le CRDI et la télédétection}

En 1987, une convention entre le CRDI et l'Université Laval permettait la réalisation d'un projet au Mali intitulé "Télédétection et planification des aménagements agraires". Ce projet est toujours en cours; son objectif principal est d'améliorer les compétences de la Direction nationale du génie rural dans le champ des applications de la télédétection destinées à l'identification et à la mise en évidence des meilleurs sites potentiels d'aménagement agraire dans la vallée du fleuve Niger. II s'agit d'abord et avant tout d'une recherche locale; le projet ne comporte pas de formation supérieure à l'Université Laval autre que des appuis académique et logistique aux chercheurs maliens par des professeurs de notre Faculté. Ce projet relève du Laboratoire de télédétection.

\section{L'Institut Atlantique de géomatique}

En juillet 1989, trois universités signaient un protocole d'entente visant la création de l'Institut Atlantique de géomatique; il s'agissait de l'Université Laval, de I'Université du Nouveau-Brunswick ainsi que de l'Université du Maine. Cet institut a pour buts de promouvoir la profession et de favoriser les échanges scientifiques entre les trois centres d'excellence.

\section{Inscription d'étudiants non canadiens}

Dès le début des années 60 , la Faculté de foresterie et de géomatique accueillait quelques étudiants non canadiens. Ces derniers provenaient surtout de l'Asie (Cambodge et Laos) et le gouvernement canadien (I'ACDI n'existait pas à cette époque) appuyait financièrement leurs programmes d'études. Il a fallu attendre le milieu des années 70 pour que la proportion de cette clientèle particulière s'accroisse de façon importante. L'arrivée massive de candidats zaïrois correspondait essentiellement à l'arrêt du projet canadien de mise en place d'un Département de foresterie au Zaïre même. Aujourd'hui, la Faculté est devenue une institution attrayante pour plusieurs pays. La présence des étudiants non canadiens se fait sentir aux trois niveaux de l'enseignement supérieur: le baccalauréat ou $1^{\text {er }}$ cycle, la maîtrise ou $2^{\text {ième }}$ cycle et le doctorat ou $3^{\text {iẻme }}$ cycle.

Grâce aux statistiques les plus récentes d'inscription (automne 1989), il est possible de tracer un portrait assez précis de la clientèle étrangère qui est en formation à la Faculté. Le tableau II indique l'importance de cette clientèle et permet de voir la distribution des étudiants par grand secteur, par région et par cycle de formation. En comparant ces données avec les inscriptions totales, on réalise qu'au $1^{\mathrm{er}}$ cycle, cette clientèle représente quelque $10 \%$ de nos étudiants, alors qu'aux $2^{\text {ième }}$ et $3^{\text {ième }}$ cycles, ce pourcentage approche 35 . Le tableau permet aussi de constater l'importance des étudiants d'Afrique francophone dans nos divers programmes de formation. Si l'on veut situer ces faits dans la perspective de la dernière décennie, on peut mentionner que de 1981 à aujourd'hui, dans les seuls programmes gradués, environ 50 candidats étrangers ont obtenu des diplômes de maîtrise ou de doctorat, la plupart venant de l'Afrique francophone. De façon générale, les organismes qui aident financièrement ces programmes de formation sont: I'ACDI, la FAO, le Gouvernement du Québec, le CRDI et I'EUMC. Un certain nombre d'étudiants sont boursiers de leurs propres pays d'origine alors que d'autres viennent à leurs frais.

L'encadrement des étudiants gradués exige un apport pluridisciplinaire adapté. La présence d'étudiants gradués non canadiens implique donc la participation continue ou ponctuelle de plusieurs professeurs de la Faculté.

\section{Présentation de conférences}

II arrive fréquemment que les professeurs de la Faculté de foresterie et de géomatique se rendent dans d'autres pays pour y prononcer des conférences. Durant les derniers 10 ans, quelque cent conférences ont ainsi été prononcées en dehors du Canada; de ce nombre, plus de la moitié environ ont été prononcées aux Etats-Unis. Ces chiffres indiquent clairement le principal pôle d'attraction pour ce volet d'intervention. Les sujets traités par ces exposés touchent tous les domaines de la foresterie et de la géomatique.

En plus de voir plusieurs de ses membres intervenir à l'extérieur du Canada, la Faculté organise à l'inverse la venue de conférenciers étrangers. Ainsi, par exemple, dans le cadre de la "Conférence Stone-Consolidated", conférence prestigieuse organisée à chaque année, la Faculté accueille 1 fois sur 2 un conférencier étranger; en plus de la conférence, la personne invitée rencontre les professeurs et chercheurs de la Faculté, les étudiants de tous les cycles et les intervenants du monde forestier québécois. II en est de même de l'Institut Atlantique de géomatique qui de façon régulière organise l'échange de conférenciers, soit issus de ses universités constituantes, soit d'autres milieux professionnels ou scientifiques.

A d'autres occasions, et de façon discontinue, certains groupes de recherche (biologie forestière, chimie du bois, etc.), reçoivent des conférenciers américains pour de courtes périodes. Le Centre de recherche en biologie forestière le fait sur une base systématique et on peut souligner qu'en moyenne, annuellement, une dizaine de personnes expertes dans l'un ou l'autre domaine de la biologie viennent à la Faculté faire bénéficier le milieu de leurs connaissances.

\section{Participation à des congrès et symposiums internationaux}

Par domaine d'intérêt, certains membres de la Faculté de foresterie et de géomatique appartiennent à des sociétés 
Tableau II. Répartition des étudiants étrangers par secteur et par cycle.

\begin{tabular}{|c|c|c|c|c|c|c|}
\hline \multirow[b]{2}{*}{ Régions d'origine } & \multicolumn{3}{|c|}{ Foresterie } & \multicolumn{3}{|c|}{ Géomatique } \\
\hline & $1^{\text {er }}$ cycle & $2^{\text {ème }}$ cycle & $3^{\text {ème }}$ cycle & $1^{\text {er }}$ cycle & $2^{\text {eme }}$ cycle & $3^{\text {ème }}$ cycle \\
\hline \multicolumn{7}{|l|}{ Afrique } \\
\hline Bénin & & & 1 & & & \\
\hline Cameroun & 2 & 1 & 1 & & & \\
\hline Zaïre & 18 & 4 & 2 & & & \\
\hline Côte d'Ivoire & & 1 & & & & 1 \\
\hline Mali & & 2 & & & & \\
\hline Maroc & & 3 & 2 & & 2 & 3 \\
\hline Rép. centrafricaine & & 2 & & & & \\
\hline Rwanda & 8 & 2 & & & & \\
\hline Sénégal & 1 & 7 & 1 & & 1 & \\
\hline Tunisie & & 3 & & & & \\
\hline Sous-total & 29 & 25 & 7 & 0 & 3 & 4 \\
\hline \multicolumn{7}{|l|}{ Amérique Latine } \\
\hline Argentine & & & & & 1 & \\
\hline Brésil & & 1 & 2 & & & \\
\hline Mexique & & 1 & 1 & & & \\
\hline Pérou & & & 1 & & & \\
\hline Sous-total & 0 & 2 & 4 & 0 & 1 & 0 \\
\hline \multicolumn{7}{|l|}{ Europe } \\
\hline Belgique & 1 & & & & & \\
\hline Bulgarie & 1 & & & & & \\
\hline France & 3 & 2 & 2 & & & \\
\hline Sous-total & 5 & 2 & 2 & 0 & 0 & 0 \\
\hline \multicolumn{7}{|l|}{ Autres } \\
\hline Chine & & & 1 & & & 1 \\
\hline Total, toutes régions & 34 & 29 & 14 & 0 & 4 & 5 \\
\hline
\end{tabular}

ou organisations dont la portée est internationale. Parmi les principales, on peut noter IUFRO (International Union of Forest Research Organizations), FPRS (Forest Products Research Society) et ISTF (International Society of Tropical Foresters). D'autres, individuellement, appartiennent à I' "American Society for Testing and Materials'" (ASTM), à la Société européenne de photo-biologie, au "Northeastern forest pathology Workshop", à la "Commission for the Application of Sciences to Agriculture, Forestry and Aquaculture" (CASAFA) ainsi qu'à la "Society of Wood Science and Technology" (SWST). Du côté de la géomatique, les membres de la Faculté appartiennent principalement à l'"American Society of Photogrammetry and Remote Sensing" (ASPRS), à l'" American Congress on Surveying and Mapping" (ACSM) ainsi qu'à l' "Urban and Regional Information Systems Association" (URISA).

Un certain nombre de professeurs membres ou non de ces sociétés, participent régulièrement à des congrès ou symposiums internationaux. En plus de ce type d'intervention, certains membres de la Faculté ont été directement impliqués dans l'organisation et la réalisation de telles rencontres internationales lorsqu'elles interviennent principalement à Québec: congrès international sur le Frankia en 1984, congrès international sur la fixation d'azote appliquée à la foresterie en 1984, première conférence des limnologistes d'expression française en 1986, congrès international de foresterie en 1984. Les trois premiers congrès avaient une portée restreinte et visaient une clientèle spécialisée. Le dernier, par contre, rejoignait une clientèle beaucoup plus large, formée des membres de l'IFC (Institut forestier du Canada), de I'OIFQ (Ordre des ingénieurs forestiers du Québec), de la SAF (Society of American Foresters) ainsi que de la IUSF (International Union of Societies of Foresters); vingt et un pays et tous les continents y étaient représentés. D'autres membres de la Faculté ont été directement impliqués dans l'organisation du congrès de la "Forest Products Research Society" (FPRS), et du congrès international de phytopathologie. Un groupe de professeurs participent actuellement à l'organisation du $3^{\text {ième }}$ séminaire international sur les sciences de la référence spatiale. D'une façon générale, l'organisation de tels congrès implique les professeurs ou chercheurs concernés, le personnel technique, le personnel de soutien, les services administratifs, et l'apport considérable des étudiants gradués des $2^{\text {ième }}$ et $3^{\text {ième }}$ cycles.

\section{Réalisation de missions techniques et professionnelles}

Les compétences professionnelles et scientifiques des professeurs de la Faculté de foresterie et de géomatique ainsi que la renommée de ses divers programmes d'enseignement et de recherche font que de façon régulière et importante plusieurs de ses membres réalisent diverses missions à l'extérieur du Canada. On peut assez facilement estimer à 50 le nombre de missions qui ont ainsi été effectuées durant les 10 dernières années; en gros ces missions ont été réalisées en Afrique $(40 \%)$, en Amérique latine (40\%) ou ailleurs dans le monde $(20 \%)$.

Les objectifs visés par ces interventions sont la plupart du temps reliés à l'un ou l'autre des éléments suivants:

- Présence d'une expertise utile à la résolution de problèmes techniques particuliers;

- Evaluation de projets exécutés ou en cours d'exécution par d'autres intervenants;

- Planification d'interventions susceptibles d'être financées par des agences internationales; 
- Exploration des possibilités ultérieures de coopération entre la Faculté de foresterie et de géomatique et d'autres organismes similaires;

- Recrutement de candidats dans le cadre de programmes de formation en foresterie ou en sciences géodésiques dont la Faculté est maître d'oeuvre:

- Implantation de programmes de recherche appropriés;

- Etude de certains secteurs forestiers;

- Développement de systèmes d'information à référence spatiale et essais de logiciels:

- Etablissement de protocoles expérimentaux pour fins de mémoires de maîtrise ou de thèses de doctorat;

- Dispense intensive de cours spécialisés durant une courte période;

Quoique la plupart de ces missions soient financées par I'ACDI, il arive que d'autres organismes interviennent aussi: CRDI, MAIQ, PNUD-FAO, etc.

\section{Organisation d'échanges}

Ce volet d'intervention comprend deux parties distinctes:

- Accueil de stagiaires non canadiens à la Faculté;

- Accueil de professeurs ou étudiants de la Faculté dans des organismes non canadiens.

Durant les dix dernières années, la Faculté, soit sur le campus même, soit dans sa forêt expérimentale, a accueilli plusieurs stagiaires dont la plus grande proportion venaient de France, du Mexique, du Zaïre, des Antilles, du Pérou, de l'Algérie ainsi que de l'Italie. La durée des stages individuels a varié de un à douze mois, pour un total cumulatif d'environ 125 mois-stage. Les organismes ayant permis la réalisation matérielle de tels échanges sont relativement variés:

- Stagiaires français: le programme d'échanges FranceQuébec, le gouvernement canadien et le CRSNG;

- Stagiaires mexicains: le programme d'échanges Canada Mexique pour jeunes spécialistes et technologues;

- Stagiaires zaïrois, péruviens et algériens: I'ACDI;

- Stagiaires italien: le ministère italien de I'Agriculture et des forêts.

Durant la même période, l'accueil à l'étranger des stagiaires de la Faculté se résume à:

- Interventions en France supportées par le programme d'échanges France - Québec: stage de trois mois pour quatre étudiants de maîtrise, stage de un mois pour un étudiant de maîtrise et stage de trois semaines pour un professeur;

- Interventions supportées par les subventions de recherche d'un professeur de la Faculté: trois séjours prolongés dans les laboratoires spécialisés en Angleterre, France et EtatsUnis pour des candidats de maîtrise et doctorat.

A ces stages libres s'ajoutent, depuis quelques années, des stages intégrés dans un cours intitulé: "Stages en foresterie internationale". Introduit depuis peu comme cours optionnel dans le programme de $1^{\text {er }}$ cycle en Aménagement des ressources forestières, ce cours crédité a jusqu'ici permis à quelque 80 étudiants d'effectuer des stages en Martinique (1987), en Guadeloupe (1988), en Guyane française (1989) et au Maroc (1990). Conçu sur la base d'échange réciproque avec les pays-hôtes, il a aussi permis à un nombre équivalent d'étudiants de l'Université des Antilles et de la Guyane et à d'autres professionnels d'effectuer au Canada un stage similaire.

En plus de ces échanges la Faculté gère un programme systématique d'échanges avec la Finlande, la Suisse et la Suède. Les résultats de cette activité font qu'en moyenne, chaque année depuis 1981, deux étudiants de la Faculté ont travaillé en Finlande et deux autres en Suède pour des périodes variant de deux à trois mois, et un autre a effectué, en Suisse, un stage de six mois. Finalement, deux étudiants finlandais ainsi que deux étudiants suédois ont travaillé au Québec, pour des périodes allant de deux à trois mois aussi. Dans ce programme d'échanges, les responsabilités de la Faculté consistent à sélectionner les candidats qui se rendront en Europe, à identifier des organismes d'accueil pour les stagiaires européens et voir à ce que l'ensemble des activités des stagiaires fonctionnent de façon optimale.

\section{Réalisation d'années sabbatiques ou sans solde}

Sept professeurs ont, ces dernières années, réalisé leurs projets sabbatiques en dehors du Canada. Les pays choisis ont été la France, les Etats-Unis, le Maroc, la Suède et la Finlande; de plus, un professeur a décidé d'oeuvrer dans plusieurs pays francophones d'Afrique. Les organismes d'accueil ont été:

- des institutions universitaires: Duke University en Caroline du Nord, Université de Paris VII, Université de Helsinki, Université suédoise des sciences agronomiques, Institut agronomique et vétérinaire Hassan II de Rabbat, Stanford University en Californie, Université du Wisconsin à Madison;

- des institutions de recherche: Institut national de la recherche agronomique de Nancy, Institut de recherches en opérations forestières de Stockholm.

La présence totale à l'étranger de ces projets sabbatiques se chiffre à 65 mois. Tous ces projets ont été appuyés financièrement par un budget spécial de l'Université Laval, à l'exception du projet au Maroc qui l'a été par l'ACDI.

Un professeur de la Faculté a de plus séjourné deux ans au Pérou grâce à l'obtention d'un congé sans solde de I'Université Laval. Cette intervention particulière s'est déroulée à l'Université La Molina à Lima au Pérou, dans le cadre d'un projet bilatéral confié par l'ACDI à l'Université de Toronto, via sa Faculté de foresterie.

Enfin, la Faculté de foresterie et de géomatique accueille, depuis peu, deux scientifiques américains, l'un avec statut de professeur subventionnel pour une période de cinq ans, et l'autre avec un statut d'attaché de recherches.

\section{Accueil de visiteurs non canadiens}

Les visiteurs non canadiens que la Faculté accueille chaque année sont nombreux et proviennent de tous les continents. Ils viennent seuls ou en groupes organisés, pour plusieurs jours mais, la plupart du temps, pour quelques 
heures; ils viendront en simple quête d'informations générales ou avec un objectif très précis. A l'image des autres volets d'intervention décrits, ces visiteurs proviennent d'abord de France, des États-Unis et de l'Afrique francophone, puis du Mexique, de l'Amérique latine et du reste de l'Europe; tout récemment, plusieurs forestiers de Chine ont de plus visité la Faculté. La plupart profitent de cette présence pour visiter le Centre de géomatique ou pour se rendre à la Station expérimentale de I'Université Laval, la Forêt Montmorency, située à $70 \mathrm{~km}$ du campus.

\section{Autres volets d'intervention}

En plus des divers volets de coopération présentés, d'autres interventions particulières ont impliqué ou impliquent encore certains membres de la Faculté. Elles sont énumérées ci-après, sans aucun ordre de présentation.

- un groupe d'étudiants gradués et un professeur participent activement à l'édition du feuillet d'information de l'"'International Union of Societies of Foresters", appelé IUSF Newsletter.

- Un professeur est co-éditeur de la revue internationale Phytocoenologia, membre du comité de rédaction des revues internationales Scripta botanica sectio $B$ phytosociologica et Documents phytosociologiques, ainsi que membre du comité de lecture de la revue internationale Braun-Blanquetia.

- Certains professeurs revisent les demandes de subvention accordées par le US National Sciences Foundation dans le domaine de la géomatique.

- Une coopération internationale en recherche implique un professeur dans deux projets en écologie réalisés en collaboration avec les universités de Georgie, de l'Yokohama et des Antilles-Guyane; ces projets sont subventionnés par le Gouvernement du Japon, par le FICU et par l'AUPELF.

- Un professeur est coordonnateur du consortium APPLE UNIVERSITE LAVAL et comme tel représente officiellement I'Université Laval dans un groupe de quelque 110 maisons d'enseignement supérieur qui ont une entente avec APPLE. La croissance de cette association est fort rapide et le niveau de ses interventions dépasse les frontières canadiennes.

- Un professeur a été durant quelques années membre actif d'un comité de I'OTAN. Cette participation s'est traduite par un très grand nombre de déplacements vers Bruxelles, siège des rencontres du dit comité, ainsi que par une participation à l'organisation d'un congrès international à Turin, Italie.

- La Faculté de foresterie et de géomatique a participé depuis le début à la mise sur pied du Centre SAHEL, centre d'excellence subventionné par l'ACDI. Un membre de la
Faculté est actuellement membre du Conseil d'administration de ce centre, en plus de cumuler la responsabilité de sa composante Formation et d'être membre de son réseau institutionnel.

- Un professeur s'est mérité deux prix d'excellence pour la qualité de ses recherches: le prix Louis-Roussel de l'Association française pour l'avancement des sciences et le prix de la Société française de photobiologie.

- Un professeur est conseiller privilégié pour l'organisme non gouvernemental CECl (Centre d'études et de coopération internationale), dont le rayonnement international est reconnu.

- La Faculté fait actuellement partie intégrante d'un réseau d'entités universitaires appliquant sur le programme des centres d'excellence de l'ACDI. Certains membres de la Faculté ont participé à la mise sur pied du projet.

- Certaines sociétés de géomatique, américaines et suisses, travaillent en collaboration étroite avec certains membres de la faculté dans des projets conjoints de recherche et développement.

- Le projet de mise sur pied d'un programme de maîtrise intitulé "Gestion des ressources renouvelables des régions tropicales' chemine actuellement aux différentes instances décisionnelles de l'Université Laval. Son acceptation permettra une formation plus adéquate pour les canadiens désireux d'oeuvrer en coopération internationale ainsi que pour la clientèle étrangère désireuse de compléter sa propre formation.

\section{Conclusion}

Ce document a voulu présenter le portrait le plus actuel des activités de coopération internationale à la Faculté de foresterie et de géomatique. Cette dernière a joué jusqu'ici un rôle primordial sur le plan international: la diversité de ses domaines de compétence, l'excellence de ses programmes de recherches, ses programmes d'enseignement et son visage francophone sont, parmi d'autres, quelques élements qui expliquent cette place privilégiée. II ne fait aucun doute que l'avenir réserve à la Faculté de foresterie et de géomatique un rôle encore plus important. La récente nomination d'un responsable facultaire de la coopération internationale s'inscrit dans cette volonté et fait suite à l'adoption, par les hautes instances de l'Université Laval, d'une nouvelle politique de coopération internationale. Les défis sont nombreux, les sollicitations fréquentes et les moyens d'intervention limités. En dépit de cette situation, la coopération internationale fait partie intégrante de la mission de l'Université Laval et la Faculté de foresterie et de géomatique, non seulement reconnaît cette responsabilité, mais désire s'y consacrer à la mesure de ses moyens. 